Preprint typeset in JHEP style - HYPER VERSION

\title{
The Delocalized Effective Degrees of Freedom of a Black Hole at Low Frequencies
}

\author{
Barak Kol \\ Racah Institute of Physics, Hebrew University \\ Jerusalem 91904, Israel \\ E-mail: barak_kol@phys.huji.ac.il
}

\begin{abstract}
Identifying the fundamental degrees of freedom of a black hole poses a longstanding puzzle. Recently Goldberger and Rothstein forwarded a theory of the low frequency degrees of freedom within the effective field theory approach, where they are relevancy-ordered but of unclear physical origin. Here these degrees of freedom are identified with near-horizon but non-compact gravitational perturbations which are decomposed into delocalized multipoles. Their world-line (kinetic) action is determined within the classical effective field theory (CLEFT) approach and their interactions are discussed. The case of the long-wavelength scattering of a scalar wave off a Schwarzschild black hole is treated in some detail, interpreting within the CLEFT approach the equality of the leading absorption cross section with the horizon area.
\end{abstract}




\section{Contents}

1. Introduction 1

2. The idea 2

3. Free scalar field in a Schwarzschild background 3

4. Discussion 5

A. Matched asymptotic expansion for long wavelength scalar cross-section 6

\section{Introduction}

Bekenstein's association of black-hole entropy with its horizon area [1] poses a deep and long-standing challenge to identify the fundamental (microscopic) degrees of freedom of a black hole. In the 90's String Theory was able to provide such a microscopical explanation of the entropy for some black holes (near-supersymmetric) in terms of a D-brane model [2, 3, 4] (and the reviews [5]). Here we shall consider the low-frequency limit of the problem which is less challenging, as it does not require Quantum Gravity, but on the other hand we shall be able to offer quite a full and satisfying picture.

In recent years an Effective Field Theory (EFT) approach to black holes was introduced by Goldberger and Rothstein [6, 7] and applied by them to dissipative effects 8]. For the purpose of describing the absorption of incoming long-wavelength radiation by the black hole they introduce certain black hole degrees of freedom in the spirit of effective field theories and with no clear physical origin. They proceed to deduce parts of the black hole effective action where these degrees of freedom appear. Finally they speculate on their origin in terms of a "hypothetical two-dimensional theory localized on the stretched horizon" (see [9] for a further discussion). An application of dissipative effect to spinning black holes appeared in [10].

This picture raises several questions

- What is the physical origin of the black hole horizon degrees of freedom, and by what procedure could they be identified?

- How could one obtain the effective action systematically?

In this note we shall solve the first question, and while we make some remarks on the second, it is mostly left for future work. We start with explaining the main idea in section 2. We proceed in section 3 to consider the concrete case of a scalar field in the background of a Schwarzschild black hole, interpreting within the EFT approach the equality of the 
leading absorption cross section with the horizon area, namely $\sigma=A{ }^{1}$ We conclude with a short summary and discussion in section 1 . Appendix A contains the details of the computation of the black hole cross-section.

\section{The idea}

Consider the full gravitational field $g_{f}$ in the background of a black hole of size $r_{0}$. The full action is given by the usual Einstein-Hilbert action

$$
S\left[g_{f}\right]=\int \sqrt{g_{f}} d^{4} x R\left[g_{f}\right] .
$$

If we furthermore consider the scattering of long wavelength waves off the black hole $(\mathrm{BH})$, then the condition for using EFT is fulfilled, namely that the typical length scale of the background is much larger than the object (the $\mathrm{BH}$ ).

Inspired by an analogy with electromagnetism [8] introduced the following world-line effective action for the black hole

$$
\begin{aligned}
S_{e f f}[x]= & -m \int d \tau+\frac{\alpha}{2} \int d \tau E^{\mu \nu} E_{\mu \nu}+\frac{\beta}{2} \int d \tau B^{\mu \nu} B_{\mu \nu}+\ldots \\
& -\int d \tau Q_{a b}^{E} E^{a b}-\int d \tau Q_{a b}^{B} B^{a b}+\ldots
\end{aligned}
$$

where $E_{\mu \nu}:=C_{\mu \alpha \nu \beta} v^{\alpha} v^{\beta}, B_{\mu \nu}:=\epsilon_{\mu \alpha \beta \rho} C_{\nu \sigma}^{\alpha \beta} v^{\rho} v^{\sigma} / 2$ are the electric and magnetic components of the Weyl tensor $C$; $a, b$ are frame ("flat space") indices rather than space-time indices; $v^{\mu}$ is the 4 -velocity of the $\mathrm{BH}$ and the constants $\alpha, \beta$ appearing in the first line are yet to be determined black-hole constants. $Q^{E}, Q^{B}$ are electric and magnetic quadruple type world-line degrees of freedom, "introducing all possible terms" in the spirit of effective field theory even though "the world-line theory is not known".

In order to learn more about the sector of the world-line theory which involves $Q^{E}, Q^{B}$ [8] analyze the two-point function. They define a function $F(\omega)$ such that

$$
\int d t e^{-i \omega t}\left\langle T Q^{E}(0) Q^{E}(t)\right\rangle \sim i F(\omega) .
$$

From known results about the long wavelength absorption cross section of Schwarzschild they deduce

$$
\operatorname{Im} F(\omega)=\frac{16}{45} G^{5} m^{6}|\omega|
$$

Then they proceed to apply these results to compute the leading absorption into a black hole moving through a curved background and an inspiraling binary.

In this paper we wish to derive the horizon degrees of freedom and their action, rather than introduce "all possible interactions". The main idea is a consequence of a closer scrutiny of the concept of "integrating out" within the set-up of Classical Effective Field Theory (CLEFT) [11] (see [12] for an application of CLEFT ideas to economize through a field re-definition the Post-Newtonian approximation).

\footnotetext{
${ }^{1}$ The author is not aware of any (asymptotically flat) black hole solution which violates this relation.
} 
In Quantum Field Theory we are normally cavalier about which fields can be integrated out, and we do not make any special requirements (except that we normally integrate out higher frequencies before lower ones). However, the classical set-up reveals a clear requirement. Assuming the full field decomposes as $g_{f}=g_{S}+\bar{g}$ where $g_{S}$ is short wavelength components and $\bar{g}$ is the boundary, or long-wavelength component, 11] defines

$$
S_{e f f}[\bar{g}] \equiv I\left[S, g_{S}\right]:=S\left[\bar{g}, g_{S}(\bar{g})\right]
$$

namely the effective action, which is the same as integrating $g_{S}$ out of $S$, is given by the full action evaluated on the full solution which is determined by the boundary conditions $\bar{g}$ and the equations of motion (this definition is consistent of course with all other definitions and avoids the Feynman path integral). This definition stresses that one is allowed to integrate out only when the remaining fields can specify a solution.

In the current set-up, the boundary zones do not consist only of the asymptotic infinity, and we must remember also the second boundary at the horizon. Accordingly, we decompose the metric field into three components

$$
g_{f}=g_{H}+g_{S}+\bar{g}
$$

where $g_{S}$ is the short component of the metric field of order $r_{0}$, while $g_{H}$ and $\bar{g}$ are longer wavelength, boundary components: $\bar{g}$ is the usual component at asymptotic infinity, while $g_{H}$ represents the component near the second boundary, namely the horizon. Accordingly, the horizon degrees of freedom, denoted by $Q$ in (2.2) are nothing but the horizon fields, where the $S O(3)$ representation (indicated by the indices $a, b)$ is naturally determined by the decomposition into spherical harmonics.

Note that so far $g_{H}$ was not considered within the EFT approach, and that is justified as long as interactions between $\bar{g}$ and $g_{H}$ did not contribute, for instance in static situations where we impose horizon regularity rather than allow for horizon degrees of freedom.

\section{Free scalar field in a Schwarzschild background}

We now proceed to present concrete formulae for our basic idea in the case of a free scalar field in the Schwarzschild background, and to apply it to gain insight into a specific physical problem.

The action is

$$
\begin{aligned}
S & =\int d V \frac{1}{2}(\partial \phi)^{2} \\
& =2 \pi \sum_{l m} \int r^{2} d r d t\left(f^{-1} \dot{\phi}^{2}-f \phi^{\prime 2}-\frac{l(l+1)}{r^{2}} \phi^{2}\right)
\end{aligned}
$$

where in the second line we used the standard Schwarzschild metric with $f(r):=1-$ $r_{0} / r$ and the Schwarzschild radius $r_{0}$; we denoted $\dot{\phi}:=\partial_{t} \phi$ and $\phi^{\prime}=\partial_{r} \phi$; and moreover we decomposed the field into spherical harmonics $\phi=\sum_{l m} \phi_{l m} Y^{l m}$ and all quadratic expressions in $\phi$ are in a condensed notation $\phi^{2} \rightarrow \phi_{l m} \phi_{l-m}$. 
An interesting and somewhat simpler problem is that of a 1d long wavelength scattering off some potential $V=V(x)$ (in flat space), but we shall not dwell on it here.

Let us consider the two boundaries: the asymptotic boundary where $r \rightarrow \infty$ and the near horizon boundary where $r \rightarrow r_{0}$. At the asymptotic boundary $f \rightarrow 1$ and we obtain

$$
S_{\infty}=2 \pi \sum_{l m} \int r^{2} d r d t\left(\dot{\phi}^{2}-\phi^{\prime 2}-\frac{l(l+1)}{r^{2}} \phi^{2}\right)
$$

which is nothing but the action for a free scalar field in $\mathbb{R}^{4}$ - a flat $4 \mathrm{~d}$ space-time.

At the near horizon boundary it is useful to change from the $r$ variable to the tortoise coordinate $r^{*}$ defined (up to a constant) through $d r^{*}:=d r / f(r)$. We obtain

$$
S_{H}=2 \pi \sum_{l m} \int r_{0}^{2} d r^{*} d t\left(\dot{\phi}^{2}-\left(\partial_{*} \phi\right)^{2}\right)
$$

where we denoted $\partial_{*}:=\partial_{r *}$ and the angular term proportional to $f l(l+1)$ vanishes since $f \rightarrow 0$ in this limit. This expression describes a free scalar field on $\mathbb{R}_{r *, t}^{2} \times \mathbb{S}_{r 0}^{2}$ - a flat $r^{*}, t$ half-plane $\left(r^{*}<0\right)$ times a horizon sphere of radius $r_{0}$, but without any kinetic term in the sphere directions.

The essential information in the effective action is encoded by the interactions between the fields on the boundaries of the two asymptotic zones, the near horizon field $\phi_{H}$ at $r^{*}=0$ and the asymptotic field $\phi_{\infty}$ at $r=0$. These interactions are equivalent to prescribing gluing boundary conditions between the two asymptotic zones and must be of the form

$$
S_{i n t}=\left.\left.2 \pi \sum_{l} c_{l} r_{0}^{l+1} \int d t \phi_{H}^{l}\right|_{r^{*}=0} \partial^{l} \phi_{\infty}^{*}\right|_{r=0} .
$$

This action is quadratic in the fields since the original action (3.1) is quadratic, and thus all equations are linear. The allowed interactions are determined by $S O(3)$ rotation invariance, where only the traceless part of $\partial^{l} \phi_{\infty}$ interacts with the spherical harmonics (the traces would have produced redundant terms anyway, since they must vanish due to the equations of motion). The power in the $r_{0}$ pre-factor is determined by dimensional analysis and $c_{l}$ are dimensionless constants to be determined through matching with a full computation in the Schwarzschild background.

An important feature of (3.4) is that the terms are relevancy-ordered in a concrete way, namely the length dimension of each term grows with the multipole number of $\phi_{\infty}$. Accordingly, the most relevant interaction comes from the monopole of the background field $\phi_{\infty}$, the next correction comes from the dipole, and so on.

\section{Low frequency cross section equals area}

The cross section for a minimally coupled scalar field (3.1) off a 4d black hole, both rotating and non-rotating, was computed in [13, 14]. Making direct use of the properties of the confluent hypergeometric function it was found there that in the low frequency limit the cross section equals the horizon area

$$
\sigma=A
$$


This result is not obvious, and somewhat surprising since usually we think of the $2 \mathrm{~d}$ projection of an object as a measure of its cross section, and not its full area embedded in $3 \mathrm{~d}$ space. As far as the author is aware no black hole of any type provides a counter-example.

Given the generality of the result one expects it to be a consequence of a more general argument, rather than a specific computation. We now proceed to show how such insight is provided by matched asymptotic expansion (MAE) or equivalently [11] CLEFT which are the appropriate tools for this limit.

For the purpose of a MAE analysis one defines three zones: asymptotic $r, r^{*} \gg 1$, intermediate $\left|\omega r^{*}\right| \ll 1$ and near horizon $r^{*} \ll-1$, together with two overlap regions. Limiting our attention to the leading monopole sector, and specifying boundary conditions such that no $\phi$ radiation is outgoing from the black hole's horizon one finds that the absorption amplitude (or transmission through the hole's gravitational potential) is given by

$$
T=2 i \omega r_{0}
$$

(see appendix A for details of the calculation).

A closer scrutiny of the MAE calculation reveals that it could be encoded à la CLEFT by a simple boundary condition requiring that $\phi_{\infty}$ is regular at $r=0$ (namely the term $1 / r$ is absent, to allow continuity of $\phi)$

$$
\left.\phi_{\infty}\right|_{r=0}=\mathcal{O}(1) .
$$

This should be interpreted as the leading interaction, or boundary condition, in the effective action (3.4).

Altogether we obtain an insight that the universal result (3.5) is a direct consequence of the effective boundary condition (3.7).

\section{Discussion}

The generalization to theories with different matter content is now conceptually clear. Of particular interest is the case where the space-time metric is the only field, and accordingly the black hole degrees of freedom at low frequency consist of near-horizon gravitational perturbations relevancy-ordered by their multipole number. For the Schwarzschild spacetime these perturbations are described by the Zerilli and Regge-Wheeler master equations, considered in the $r^{*} \rightarrow-\infty$ limit. The lowest multipole in the expansion is the quadrupole, of course, rather than the monopole as for the scalar field.

We would like to stress the general features of the emerging picture. At low frequencies the black hole degrees of freedom were identified with near horizon fields, namely fields on $\mathbb{R}_{r *, t}^{2} \times \mathbb{S}_{r 0}^{2}$, where the first factor is half $\left(r^{*}<0\right)$ a flat $2 \mathrm{~d}$ space-time, and the second factor is the horizon sphere of radius $r_{0}$. This space is non-compact (even though it does not include the asymptotic infinity) due to the usual reason that it takes infinite asymptotic time $t$ to reach the horizon. The origin of the degrees of freedom is simply the available fields, and in particular, since the black hole is made of curved space-time its inherent degrees of freedom are near-horizon gravitational waves. The degrees of freedom are relevancyordered according to their multipole number and in this sense they are delocalized over the 
horizon. One wonders: which of these features, if any, carries over to the fundamental, short-distance degrees of freedom?

\section{Acknowledgements}

I would like to thank the members of our group for comments on a talk. This research is supported by The Israel Science Foundation grant no 607/05, DIP grant H.52, EU grant MRTN-CT-2004-512194 and the Einstein Center at the Hebrew University.

\section{A. Matched asymptotic expansion for long wavelength scalar cross-section}

We wish to compute the absorption (or transmission) amplitude for an $l=0$ low frequency scalar wave impinging on a Schwarzschild black hole.

The full equation derived from (3.1) is

$$
-\frac{1}{r^{2}} \partial_{r} f(r) r^{2} \partial_{r} \phi=\omega^{2} \phi
$$

where $f=1-r_{0} / r$, we work in the limit $\omega r_{0} \ll 1$ and henceforth we use units where $r_{0}=1$. The boundary condition is that there are no waves outgoing from the horizon, namely

$$
\begin{array}{lrl}
\phi & \sim T \exp \left(i \omega r^{*}\right) & \text { for } r^{*} \rightarrow-\infty \\
\phi & \sim 1 \cdot \exp (i \omega r) / r+R \exp (-i \omega r) / r & \text { for } r \rightarrow+\infty
\end{array}
$$

where $R, T$ are unknown reflection and transmission coefficients, which depend on $\omega$, and our objective is to determine $T$ for small $\omega$.

Our limit is appropriate for the method of matched asymptotic expansion, since the typical length of the background field, $1 / \omega$, is much larger than $r_{0}$ the size of the black hole. In order to solve this equation we divide the $r^{*}$ axis into three zones whose overlap grows in the limit

- Near horizon zone $r^{*} \ll-1$,

where we approximate $r \simeq 1$ and the equation (A.1) becomes $-\partial_{*}^{2} \phi=\omega^{2} \phi$ whose solutions are $\exp \left( \pm i \omega r^{*}\right)$.

- Intermediate zone $\left|\omega r^{*}\right| \ll 1$,

where we approximate $\omega \simeq 0$ and the equation (A.1) becomes $-\frac{1}{r^{2}} \partial_{r} f(r) r^{2} \partial_{r} \phi=0$ whose solutions are $1, \log (f)$.

- Asymptotic zone $r \simeq r^{*} \gg 1$,

where we approximate $f \simeq 1$ and the equation (A.1) becomes $-\frac{1}{r^{2}} \partial_{r} r^{2} \partial_{r} \phi=\omega^{2} \phi$ whose solutions are $\exp ( \pm i \omega r) / r$.

Solution. The boundary condition (A.2) is imposed after substituting $\phi \rightarrow T \phi$ for later convenience such that in the near horizon zone we have

$$
\phi \simeq \exp \left(i \omega r^{*}\right)
$$


We proceed to match over the overlap of the near horizon and intermediate zones, namely for $-1 / \omega \ll r^{*} \ll-1$ where the equation (A.1) becomes $-\partial_{*}^{2} \phi=0$ and the solutions are $1, r^{*}$ to obtain the solution in the intermediate zone

$$
\phi \simeq 1+i \omega(1+\log f)
$$

Next we match over the overlap of the intermediate and asymptotic zones, namely for

$1 \ll r \ll 1 / \omega$ where the equation (A.1) becomes $-\frac{1}{r^{2}} \partial_{r} r^{2} \partial_{r} \phi=0$ and the solutions are $1,1 / r$ to obtain the solution in the asymptotic zone

$$
\phi \simeq \frac{1}{2}\left(\frac{1}{i \omega}+1-i \omega\right) \exp (i \omega r) / r-\frac{1}{2}\left(\frac{1}{i \omega}+1-i \omega\right) \exp (-i \omega r) / r
$$

By comparing with the asymptotic form $\phi \sim(1 / T) \exp (i \omega r) / r+(R / T) \exp (-i \omega r) / r$ we obtain the scattering amplitudes, and in particular, the absorption (or transmission) amplitude to leading order in $\omega$ is

$$
T=2 i \omega r_{0}
$$

\section{References}

[1] J. D. Bekenstein, "Black holes and entropy," Phys. Rev. D 7, 2333 (1973).

[2] L. Susskind and J. Uglum, "Black hole entropy in canonical quantum gravity and superstring theory," Phys. Rev. D 50, 2700 (1994) [arXiv:hep-th/9401070].

[3] A. Strominger and C. Vafa, "Microscopic Origin of the Bekenstein-Hawking Entropy," Phys. Lett. B 379, 99 (1996) [arXiv:hep-th/9601029].

[4] C. G. Callan and J. M. Maldacena, "D-brane Approach to Black Hole Quantum Mechanics," Nucl. Phys. B 472, 591 (1996) [arXiv:hep-th/9602043].

[5] A. W. Peet, "The Bekenstein formula and string theory (N-brane theory)," Class. Quant. Grav. 15, 3291 (1998) [arXiv:hep-th/9712253]. S. D. Mathur, "The quantum structure of black holes," Class. Quant. Grav. 23, R115 (2006) [arXiv:hep-th/0510180].

[6] W. D. Goldberger and I. Z. Rothstein, "An effective field theory of gravity for extended objects," Phys. Rev. D 73, 104029 (2006) [arXiv:hep-th/0409156].

[7] W. D. Goldberger, "Les Houches lectures on effective field theories and gravitational radiation," arXiv:hep-ph/0701129.

[8] W. D. Goldberger and I. Z. Rothstein, "Dissipative effects in the world-line approach to black hole dynamics," Phys. Rev. D 73, 104030 (2006) [arXiv:hep-th/0511133].

[9] W. D. Goldberger and I. Z. Rothstein, "Towers of gravitational theories," Gen. Rel. Grav. 38, 1537 (2006) [Int. J. Mod. Phys. D 15, 2293 (2006)] [arXiv:hep-th/0605238].

[10] R. A. Porto, "Absorption Effects due to Spin in the Worldline Approach to Black Hole Dynamics," arXiv:0710.5150 [hep-th].

[11] B. Kol and M. Smolkin, "Classical Effective Field Theory and Caged Black Holes," Phys. Rev. D 77, 064033 (2008) [arXiv:0712.2822 [hep-th]].

[12] B. Kol and M. Smolkin, "Non-Relativistic Gravitation: From Newton to Einstein and Back," arXiv:0712.4116 [hep-th]. 
[13] D. N. Page, "Particle Emission Rates From A Black Hole: Massless Particles From An Uncharged, Nonrotating Hole," Phys. Rev. D 13, 198 (1976).

[14] A. A. Starobinsky and S. M. Churilov, Zh. Eksp. Teor. Fiz. 65, 3 (1973) [Sov. Phys. - JETP 38, 1 (1974)]. 